\title{
Found: High Surface Brightness Compact Galaxies
}

\author{
M.J. Drinkwater
}

Physics, University of New South Wales, Sydney 2052, Australia

S. Phillipps, J.B. Jones

Physics, University of Bristol, Tyndall Avenue, Bristol BS8 1TL, UK

M.D. Gregg

IGPP, Lawrence Livermore Lab., L-413, Livermore, CA 94550, USA

Q.A. Parker

Anglo-Australian Observatory, Coonabarabran, NSW 2357, Australia

R.M. Smith, J.I. Davies

Physics 83 Astronomy, University of Wales, Cardiff CF2 $3 Y B, U K$

E.M. Sadler

School of Physics, University of Sydney, NSW 2006, Australia

\begin{abstract}
We are using the $2 \mathrm{dF}$ spectrograph to make a survey of all objects ("stars" and "galaxies") in a $12 \mathrm{deg}^{2}$ region towards the Fornax cluster. We have discovered a population of compact emission-line galaxies unresolved on photographic sky survey plates and therefore missing in most galaxy surveys based on such material. These galaxies are as luminous as normal field galaxies. Using $\mathrm{H} \alpha$ to estimate star formation they contribute at least an additional $5 \%$ to the local star formation rate.
\end{abstract}

\section{Introduction}

Most galaxy surveys only detect galaxies with a limited range of surface brightness (SB): the difficulty of detecting low SB (LSB) galaxies is well-accepted (Impey, Bothun \& Malin 1988, Ferguson \& McGaugh 1995) but at the other extreme, it has been argued that there is no selection against high SB (HSB) galaxies (Allen \& Shu 1979). This was based on a small sample of bright galaxies, but the conclusion has since been applied to nearly all galaxy samples based on photographic sky surveys. Several groups have in fact detected compact galaxies unresolved on photographic survey plates, often termed compact emission-line galaxies (CELGs). Many have been found in optical QSO surveys (Guzman et al. 1998, Stobie et al. 1997, Boyle et al. 1998). Similar galaxies have been found 

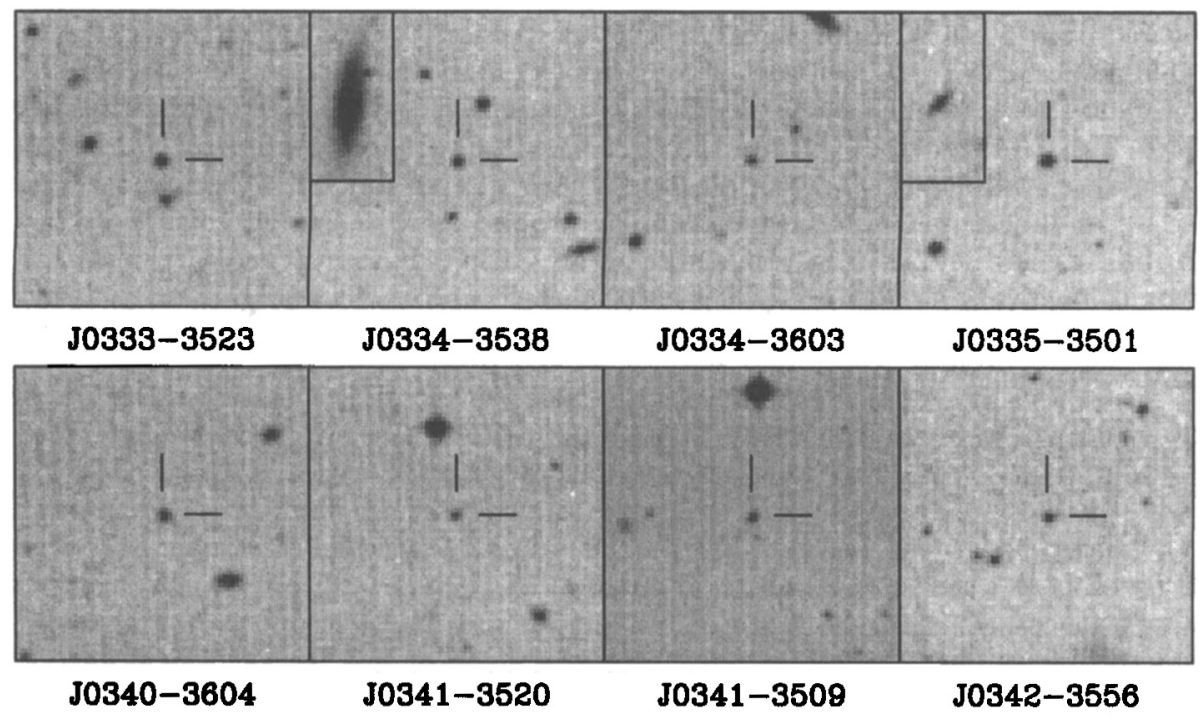

Figure 1. $\quad B_{J}$-band images of the new compact field galaxies. Each is $2^{\prime}$ across. The insets in the images of J0334-3538 and J0335-3501 show two normal $\left(M^{*}\right)$ galaxies at the same distances.

in the University of Michigan emission line galaxy survey (Salzer et al. 1989) and among HII galaxies (Terlevich et al. 1991). Unfortunately, none of these are from simple flux- or size-limited samples so they cannot be used to determine the true extent of the compact galaxy population.

In this paper we present the detection of a new population of compact galaxies based on initial results of The Fornax Spectroscopic Survey (see Drinkwater, Phillipps \& Jones, this volume), a complete survey of all objects in a region of sky centred on the Fornax cluster, being carried out with the $2 \mathrm{dF}$ multi-object spectrograph on the AAT. Of 2947 "stars" and 1290 "galaxies" in the central $2 \mathrm{dF}$ field down to $B_{J}=19.7$, we successfully observed $1249(42 \%)$ and 1250 (97\%) respectively. Among the "stars" we found a number of objects with HII region-type spectra at redshifts of $10-50,000 \mathrm{~km} / \mathrm{s}$ making them field galaxies well beyond the Fornax cluster $(1500 \mathrm{~km} / \mathrm{s})$. These results are presented in more detail by Drinkwater et al. (1998).

\section{Properties of the New Compact Galaxies Galaxies}

We present images of the new compact galaxies in Figure 1. The new CELGs have similar absolute magnitudes and distances to "normal" field galaxies, but were classified as stars both by eye and in the APM catalogue. For a typical seeing in survey plates (2") this is equivalent to scale sizes less than about 1", or physical scale sizes $0.6-2.2 \mathrm{kpc}\left(H_{0}=75 \mathrm{~km} \mathrm{~s}^{-1} \mathrm{Mpc}^{-1}\right)$. These upper limits are less than is typical of the local spiral galaxy population (de Jong 1996). However, these are not dwarfs in terms of their luminosities which are within a 
factor 10 or so of $L^{*}$; indeed two of them exceed $L^{*}$. This is clearly because of their high surface brightnesses. If we take the limit of 1 " to be the scale size of an exponential profile, then the magnitudes imply central surface brightnesses 19.5-21.8 Bmag/arcsec ${ }^{2}$, from 1 to 5 times brighter than "normal" spirals or irregulars (van der Kruit 1987, de Jong 1996). Interestingly, if we extrapolate to larger distances, the CELGs have similar properties to the small galaxies in the Hubble Deep Field. For instance, one of our CELGs placed at redshifts $z=0.2-1$ would have very similar scale lengths $(0.1-0.2$ ") and surface brightness to those measured by Jones, Disney \& Phillipps (1998) for disk-like galaxies in the HDF.

\section{Significance of the New Population}

The discovery of these CELGs poses several questions, notably what fraction of all galaxies has been missed? At redshifts less than $55000 \mathrm{~km} / \mathrm{s}$ we observed 600 resolved objects (galaxies): about 300 of these exhibited strong emission line features. Within the same distance and magnitude limits we found seven new CELGs. These therefore constitute about $1 \%$ of the observed local galaxy population, but have been missed by existing surveys. All the new compacts have strong $\mathrm{H} \alpha$ emission, so they represent twice this fraction of emission line galaxies $(2 \%)$. We can go one step further and consider their contribution to the local star formation rate as $\mathrm{H} \alpha$ emission is a measure of recent star formation (Tresse $\&$ Maddox 1998). Our spectra are not flux-calibrated and the 2" apertures only partially sample the flux from resolved objects. However the equivalent width of the emission lines is proportional to the absolute flux in the emission lines of the different galaxies to first order if we assume that the galaxies all come from the same distribution of intrinsic magnitudes and distances as shown above. On this basis the seven new compact galaxies contribute an extra. $5 \%$ of star formation rate over that in the whole sample of 600 . As we have surveyed less than half of the unresolved objects, these numbers are likely to double in the final analysis.

\section{References}

Allen R.J., Shu F.H., 1979, ApJ, 227, 67

Boyle B.J., et al., 1998, Phil. Trans. R. Soc. Lond. A, in press

Drinkwater, M.J., et al., ApJ, submitted

Ferguson H.C., McGaugh S.S., 1995, AJ, 440, 470

Guzman R., Jangren A., Koo D., Bershady M., Simard L. 1998, ApJ, 495, L13

Impey C., Bothun G., Malin D., 1988, ApJ, 330, 634

Jones J.B., Disney M.J., Phillipps S., 1998, MNRAS, submitted

de Jong, R.S. 1996, A\&A, 313, 45

van der Kruit P.C., 1987, A\&A, 173, 59

Salzer J.J., MacAlpine G.M., Boroson T.A., 1989, ApJS, 70, 479

Stobie et al., 1997, MNRAS, 287, 848

Terlevich R., Melnick J., Masegosa J., Moles M., Copetti, 1991, A\&AS, 91, 285

Tresse L., Maddox S.J., 1998, ApJ, 495, 691 